\section{Effects of Long-Term, Low-Dose Macrolide Treatment on Pseudomonas aeruginosa PAO1 Virulence Factors In Vitro}

\section{Abstract}

Pseudomonas aeruginosa ( $P$. aeruginosa) is a common cause of chronic airway infections in patients with pulmonary disorders such as diffuse panbronchiolitis (DPB) and cystic fibrosis (CF). Long-term, low-dose macrolide treatment has markedly increased long-term survival of patients with DPB. Consequently, researchers are interested in using macrolides to treat CF patients. Previous studies have demonstrated that macrolides influence $P$. aeruginosa virulence. However, most studies evaluated the regulatory effects of macrolides on $P$. aeruginosa virulence factors after treatment over a short period ( $<48$ hours). In this study, we subcultured $P$. aeruginosa PAO1 for 2 to 18 months in the presence of lowdose macrolides and evaluated antibiotic minimum inhibitory concentrations (MICs), bacterial growth, and virulence factors, including various motilities, biofilm formation, and production of rhamnolipids, total protease, elastase, and pyocyanin. The production of many virulence factors gradually decreased with macrolide exposure, and some were maximally affected after only 2 months. Despite an initial inhibition after treatment with macrolides, treated bacteria later exhibited increased biofilm formation compared to untreated controls. These findings suggest that low-dose macrolide therapy for chronic airway infection should be administered for at least 2 months to achieve optimal effects against $P$. aeruginosa.

Keywords: Macrolides; Pseudomonas aeruginosa; Virulence factor

\author{
Suzuki $\mathrm{S}^{1 *}$, Mano $\mathrm{Y}^{2}$, \\ Fujitani $K^{1,3}$ and Furuya $\mathbf{N}^{1,2}$ \\ 1 Graduate School of Health Care \\ Science, Bunkyo Gakuin University 19-1, \\ Mukogaoka 1-chome, Bunkyo-ku, Tokyo \\ 113-8668, Japan \\ 2 Department of Clinical Laboratory \\ Medicine, Bunkyo Gakuin University 4-1, \\ Mukogaoka 2-chome, Bunkyo-ku, Tokyo \\ 113-0023, Japan \\ 3 Department of Occupational Therapy, \\ Bunkyo Gakuin University 1196 \\ Kamekubo, Fujimino-shi, Saitama 356- \\ 8533, Japan
}

\section{*Corresponding author: Shusaku S}

\section{syusaku0615@gmail.com}

Graduate School of Health Care Science, Bunkyo Gakuin University 19-1, Mukogaoka 1-chome, Bunkyo-ku, Tokyo 113-8668, Japan.

Tel: $+81-3-3811-0441$

Fax: $+81-3-3811-0439$

Citation: Suzuki S, Mano Y, Fujitani $K$ and Furuya N (2017) Effects of LongTerm, Low-Dose Macrolide Treatment on Pseudomonas aeruginosa PAO1 Virulence Factors In Vitro. Arch Clin Microbiol. Vol. 8 No. $4: 50$.

Pseudomonas aeruginosa ( $P$. aeruginosa), a ubiquitous Gramnegative, rod-shaped bacterium, is an obligate aerobe with minimal nutritional requirements. It is often found in moist environments including soil, water, and sewage [1,2], and is an important opportunistic pathogen in humans, which primarily contributes to hospital-acquired bacterial infections in immunocompromised hosts [2-4]. Moreover, $P$. aeruginosa is not only the leading cause of chronic lung infections in patients with panbronchiolitis (DPB) and cystic fibrosis (CF), it is also a frequent cause of exacerbations in individuals with advanced chronic obstructive pulmonary disease (COPD) [5]. P. aeruginosa has a high intrinsic resistance to most antibiotics $[6,7]$ and possesses a large array of virulence factors, such as flagella, pili, lipopolysaccharide, the formation of biofilms, and the production of elastase, protease, and pyocyanin [8]. Repeated prolonged courses of broad-spectrum antibiotics lead to the selection of increasingly antibiotic-tolerant and resistant strains [9].

DPB is a chronic infection of the lower respiratory tract common among the Japanese people, with a persistent $P$. aeruginosa infection and sustained neutrophil retention in the airways [10]. In addition, DPB was an inflammatory disease of the airways with a high rate of mortality despite treatment with a combination of antibiotics and the use of supportive therapy such as oxygen administration [11]. Recently, long-term, low-dose macrolide treatment for chronic $P$. aeruginosa respiratory infections was 
shown to alter the clinical course of DPB [11]. Macrolides are common antibiotics used in patients with respiratory infections; however, they were thought to have weak or no activity against $P$. aeruginosa since the maximum concentrations of macrolides in serum and sputum are below minimum inhibitory concentrations (MICs) and fail to inhibit the proliferation of $P$. aeruginosa [12]. Nevertheless, the introduction of long-term macrolide therapy for chronic $P$. aeruginosa airway infection in DPB has resulted in dramatic improvements in survival, with 5-year survival rates increasing from 63 to $92 \%$ [13].

Previous studies demonstrated that the therapeutic benefits of macrolides against $P$. aeruginosa are mediated by inhibition of adherence, production of virulence factors, and formation of biofilm, as well as inhibition of the quorum-sensing circuitry, which leads to reduced virulence factor production [14-17]. However, most studies evaluated regulation of $P$. aeruginosa virulence factors after treatment with macrolides for a short period ( $<48$ hours), there have been no report that investigating the effect of long-term, low-dose exposure of macrolide against $P$. aeruginosa. In this study, we evaluated $P$. aeruginosa subcultured for 2 to 18 months in the presence of low-dose macrolides to investigate the appropriate period of long-term, low-dose macrolide treatment; we investigated the effects of long-term exposure on MICs, bacterial growth, and virulence factors, including motility, biofilm formation, and production of rhamnolipids, total protease, elastase, and pyocyanin.

\section{Materials and Methods}

\section{Bacterial strains}

In order to mimic long-term, low-dose macrolide treatment in vitro, erythromycin and clarithromycin, designated as the first or second-choice drug of DPB treatment, were used [18]. We determined the appropriate macrolide concentration by referencing the maximum drug concentration ( $\mathrm{Cmax}$ ) at the time of oral administration in the macrolide treatment studies [1820]. $P$. aeruginosa PAO1 was repeatedly subcultured 76 times over 18 months on Mueller-Hinton agar (BD Diagnostics, Sparks, $\mathrm{MD}$, USA) containing $1.6 \mathrm{\mu g} / \mathrm{mL}$ erythromycin (Wako Pure Chemical Industries, Osaka, Japan) or $0.8 \mu \mathrm{g} / \mathrm{mL}$ clarithromycin (LKT Laboratories, Saint Paul, MN, USA). P. aeruginosa PAO1 that subcultured in the presence of low-dose macrolides were frozen at 2 month intervals, and we generated strains with long-term, low-dose macrolide exposure (exposure time: 2, 4, $6,8,10,12,14,16$ and 18 month). Non-exposure $P$. aeruginosa PAO1 (original strain) to macrolide was used as a strain with a macrolide exposure period of 0 month.

\section{Bacterial growth assay}

Aliquots $(20 \mu \mathrm{L})$ of bacterial solutions were adjusted to $1.5 \times 10^{7}$ colony-forming units (CFU)/mL with sterile physiological saline, then inoculated into $6.0 \mathrm{~mL}$ of LB broth (BD Diagnostics, Sparks, MD, USA) and cultured with shaking (130 rpm) at $35^{\circ} \mathrm{C}$ for 24 hours. $10 \mu \mathrm{L}$ of the culture solution were diluted from 10 -fold to $10^{9}$-fold at two hour intervals, and each dilution $(10 \mu \mathrm{L})$ were plating the LB agar (BD Diagnostics, Sparks, MD, USA) and cultured overnight. Bacterial growth was determined by counting CFU.

\section{Antimicrobial susceptibility testing}

Antimicrobial susceptibility of all $P$. aeruginosa strains was evaluated by microdilution, in accordance with the Clinical and Laboratory Standards Institute (CLSI) M100-S25 standard [21]. MICs were determined for the following antibiotics: ceftazidime (Tokyo Chemical Industry, Tokyo, Japan); imipenem, meropenem and clarithromycin (LKT Laboratories, Saint Paul, MN, USA.); and gentamicin, amikacin, erythromycin, and ciprofloxacin (Wako Pure Chemical Industries, Osaka, Japan).

\section{Motility assays}

Swimming: $P$. aeruginosa ( $10 \mu \mathrm{L}$ aliquots adjusted to $10^{9} \mathrm{CFU} /$ $\mathrm{mL}$ ) were inoculated in the center of swim plates $(1.0 \%$ (vol/vol) tryptone (Kanto Chemical, Tokyo, Japan), 0.5\% (vol/vol) $\mathrm{NaCl}$ (Wako Pure Chemical Industries, Osaka, Japan), and 0.3\% agar (Kanto Chemical, Tokyo, Japan)), and incubated for 18 hours at $30^{\circ} \mathrm{C}$. Motility was assessed by observation of the circular turbid zone formed by bacteria migrating away from the point of inoculation [22].

Swarming: A $10-\mu \mathrm{L}$ aliquot of the inoculum was point-placed on the agar surface of the semisolid swarming plate $(0.8 \%$ Nutrient Broth (BD Diagnostics, Sparks, MD, USA), 0.5\% (vol/vol) glucose (Wako Pure Chemical Industries, Osaka, Japan), and 0.5\% agar), and the plates were incubated at $35^{\circ} \mathrm{C}$ for 24 hours. Bacterial halos were measured as an indication of swarming motility [22].

Twitching motility: We used $2.0 \%$ LB broth solidified with $1 \%$ agar for the twitching assays. Strains were stab-inoculated with a sharp sterile toothpick through a thin LB agar layer (1\%, wt/ vol), such that the toothpick reached the bottom of the plate. After incubation for 48 hours at $37^{\circ} \mathrm{C}$, a hazy zone of growth at the interface between the agar and the bottom of the plate was observed [22].

\section{Rhamnolipid production assay}

The medium composition of the rhamnolipid plate was based on $\mathrm{M} 9$ salts [23] supplemented with $0.2 \%$ (vol/vol) glucose, 2 $\mathrm{mM} \mathrm{MgSO}_{4}$ (Kanto Chemical, Tokyo, Japan), 0.0005\% (vol/vol) methylene blue (Wako Pure Chemical Industries, Osaka, Japan), $0.02 \%$ (vol/vol) cetyltrimethylammonium bromide (Wako Pure Chemical Industries, Osaka, Japan), and trace elements, and solidified with $2 \%$ agar. Glutamic acid (Wako Pure Chemical Industries, Osaka, Japan), used as the sole nitrogen source, was added to a final concentration of $0.05 \%$. Aliquots of the bacterial solutions $\left(20 \mu \mathrm{L}\right.$, adjusted to $1.5 \times 10^{7} \mathrm{CFU} / \mathrm{mL}$ with sterile physiological saline) were inoculated into $6.0 \mathrm{~mL}$ of LB broth and cultured with shaking at $35^{\circ} \mathrm{C}$ for 18 hours. We inoculated $2 \mu \mathrm{L}$ of culture solutions into rhamnolipid plates, which were cultured at $35^{\circ} \mathrm{C}$ for 24 hours, then kept for 72 hours at $25^{\circ} \mathrm{C}$ until a blue halo appeared around the colonies, indicating the production of rhamnolipids [24].

\section{Biofilm formation assay}

$P$. aeruginosa strains were grown in $5.0 \mathrm{~mL}$ of LB broth overnight at $35^{\circ} \mathrm{C}$ with shaking. Aliquots of culture solution $(180 \mu \mathrm{L}$, adjusted to $1.5 \times 10^{7} \mathrm{CFU} / \mathrm{mL}$ with LB broth) were inoculated into 96 -well 
microtiter plates and cultured at $35^{\circ} \mathrm{C}$ for 24 hours, then the microtiter plates were stained with $20 \mu \mathrm{L}$ of $1.0 \%$ (wt/vol) crystal violet (Kanto Chemical, Tokyo, Japan) for 10 minutes at room temperature and washed five times with PBS. After addition of $200 \mu \mathrm{L}$ of $95 \%$ ethanol to each well, the adsorbed dye was quantified using the optical density (OD) reading at $570 \mathrm{~nm}$ [25].

\section{Total protease assay}

The total proteolytic activity of bacterial supernatants was determined with the Remazol Brilliant Blue R-Hide assay (SigmaAldrich, Saint Louis, MO, USA), as previously described [26]. Briefly, we inoculated $20 \mu \mathrm{L}$ of bacterial solution (adjusted to 1.5 $\times 10^{7} \mathrm{CFU} / \mathrm{mL}$ ) into $6.0 \mathrm{~mL}$ of LB broth, which was incubated with shaking at $35^{\circ} \mathrm{C}$ for 18 hours. The culture solution was centrifuged at $2300 \times g$ for 15 minutes and the resulting supernatant was sterilized with a $0.22-\mu \mathrm{m}$ filter (Toyo Roshi Kaisha, Tokyo, Japan). Subsequently, $2.0 \mathrm{~mL}$ of the culture supernatants was diluted with $1.0 \mathrm{~mL}$ of $10 \mathrm{mM}$ Tris $\mathrm{HCl}, \mathrm{pH} 7.5$, and added to $3.0 \mathrm{mg}$ of Remazol Brilliant Blue R-Hide. The mixture was incubated at $37^{\circ} \mathrm{C}$ for 1 hour with shaking. The absorbance of the supernatant was measured at $595 \mathrm{~nm}$ to determine total protease activity.

\section{Elastase assay}

The elastase activity of bacterial supernatants was determined with the Elastin-Congo Red assay (Sigma-Aldrich, Saint Louis, MO, USA), as previously described [27]. An aliquot of $0.5 \mathrm{~mL}$ of culture supernatant was diluted 1 in 2 in $100 \mathrm{mM}$ Tris $\mathrm{HCl}, \mathrm{pH} 7.5$, and added to $10 \mathrm{mg}$ of Elastin-Congo red substrate. The mixture was incubated at $37^{\circ} \mathrm{C}$ for 6 hours with shaking. Undissolved substrate was removed by centrifugation at $2300 \times g$ for 5 minutes. The absorbance of the supernatant was measured at $492 \mathrm{~nm}$ to determine the elastase activity.

\section{Pyocyanin assay}

The pyocyanin assay was based upon the absorbance of excreted pyocyanin in acidic solution at $520 \mathrm{~nm}$ [28]. A $3.0 \mathrm{~mL}$ aliquot of bacterial supernatant was mixed with $1.8 \mathrm{~mL}$ of chloroform and incubated at $25^{\circ} \mathrm{C}$ for 3 hours. The pyocyanin from the chloroform phase was extracted into $1.0 \mathrm{~mL}$ of $0.2 \mathrm{M} \mathrm{HCl}$ (Wako Pure Chemical Industries, Osaka, Japan); the resulting pink to deep red color indicated the presence of pyocyanin. The absorbance was measured at $520 \mathrm{~nm}$.

\section{Result}

\section{Effects of long-term, low-dose macrolide treatment on MICs and bacterial growth}

Long-term, low-dose macrolide treatment of $P$. aeruginosa PAO1 had no effect on the MICs of any of the antibiotics used (data not shown). The results of the bacterial growth rate are shown in Figure 1. Treatment with macrolides did not affect the growth of $P$. aeruginosa $\mathrm{PAO}$.

\section{Correlation of virulence factors with macrolide}

Although long-term, low-dose macrolide treatment did not alter the $P$. aeruginosa PAO1 swimming phenotype, it appeared to reduce swarming and twitching motility (Figure 2). At all exposure durations tested, erythromycin decreased the swarming of $P$. aeruginosa $\mathrm{PAO} 1$ (Figure 2B). Clarithromycin decreased swarming of strains treated for 2 or more months. Twitching motility gradually decreased with longer periods of macrolide exposure (Figure 2C). As shown in Figure 3, rhamnolipid production decreased with longer exposure to erythromycin. Similar findings were also obtained with $P$. aeruginosa PAO1 treated with longterm, low-dose clarithromycin.

Total protease production decreased with prolongation of the exposure period to erythromycin (Figure 4A). In the strains exposed to clarithromycin for up to 12 months, total protease production was equivalent to that of the original strain. However, we observed a decrease in the production of total protease after 14 months.

Elastase production also decreased with prolonged exposure to macrolides (Figure $4 \mathbf{B}$ ). In addition, macrolide treatment
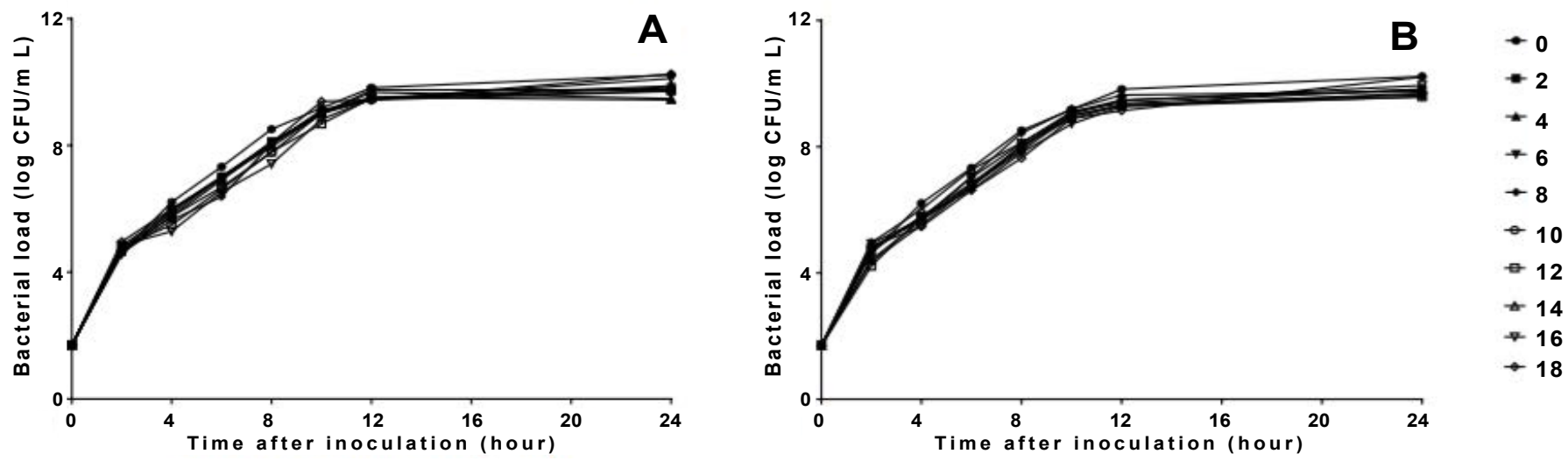

Figure 1 Bacterial growth rate of PAO1 exposed to erythromycin (A) and clarithromycin (B). Symbols indicate length of exposure in months. Growth curve determinations were repeated three times and graphs show results from one representative experiment. 

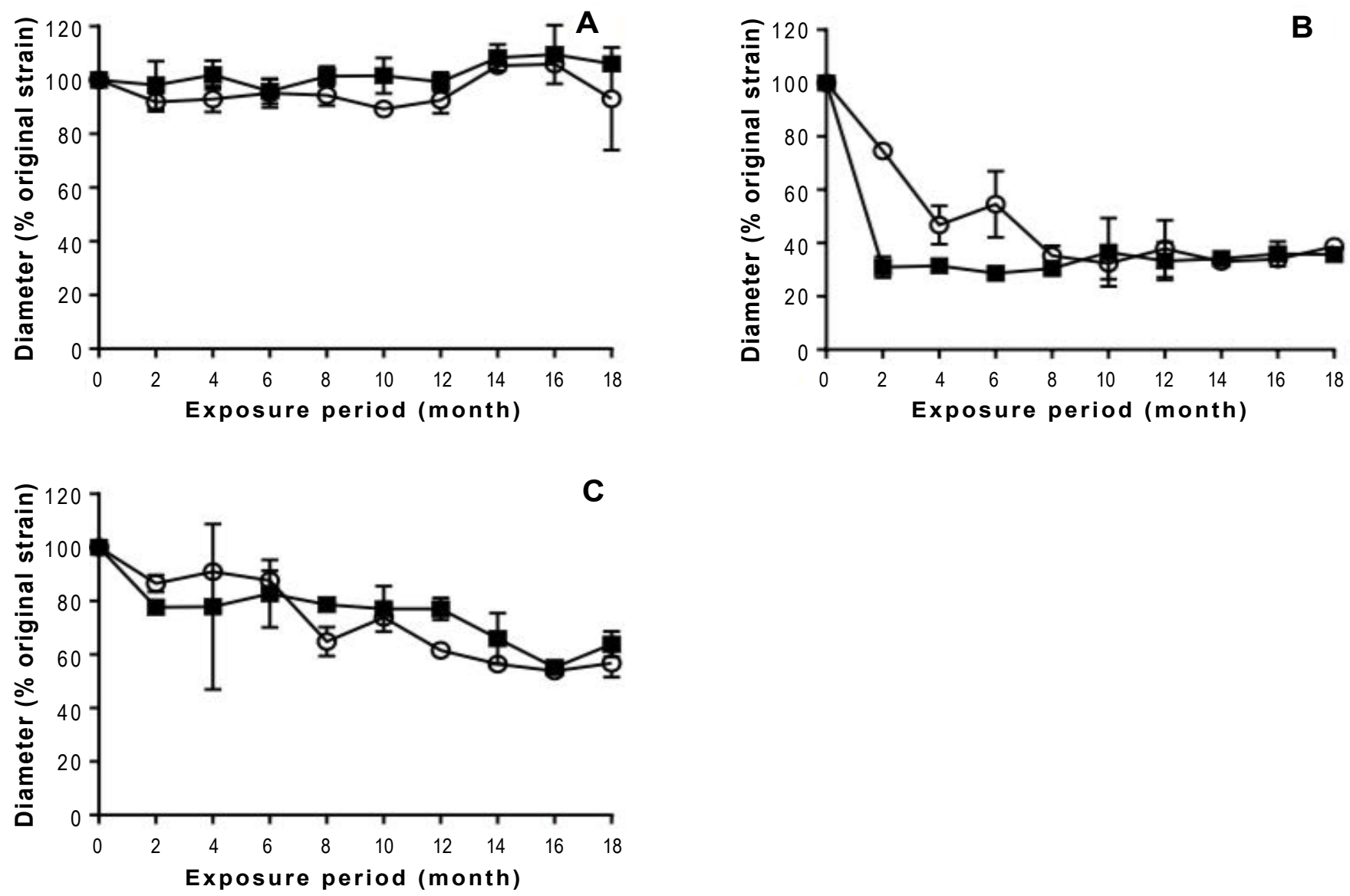

Figure 2 Motility such as swimming (A), swarming (B), and twitching (C) of PAO1 exposed to erythromycin (O) and clarithromycin ( $\mathbf{\square})$. Results are normalized to PAO1 with no macrolide exposure (original strain), and graphs display mean \pm SD of three independent experiments.

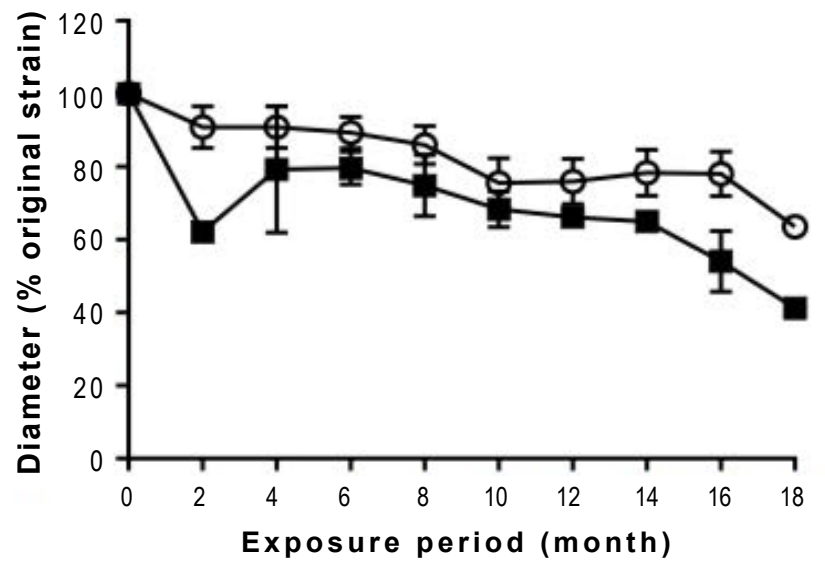

Figure 3 Rhamnolipid production by PAO1 exposed to erythromycin (O) and clarithromycin ( $\square$ ). Results were normalized to PAO1 with no macrolide exposure (original strain), and graphs display mean \pm SD of three independent experiments.

decreased pyocyanin production in the $P$. aeruginosa PAO1 strains treated for 2 or more months (Figure $4 \mathrm{C}$ ).
Exposure to macrolides promoted biofilm formation after an initial period of slight inhibition (Figure 5). Erythromycin exposure tended to inhibit the ability of PAO1 to form biofilms until the 6 month, after which it increasingly promoted biofilm formation. Similarly, biofilm formation was inhibited by clarithromycin exposure until month 12 , after which the formation increased with the exposure period.

\section{Discussion}

In this study, we investigated the time-dependent effects of low-dose macrolide treatment on several $P$. aeruginosa PAO1 characteristics. Many studies have previously reported the effects of macrolides on $P$. aeruginosa [14-17]. However, those studies examined $P$. aeruginosa after short-term macrolide exposure in vitro, and there have been no reports on the timedependent effects of long-term macrolide exposure. Therefore, we investigated changes in $P$. aeruginosa PAO1 virulence factors after long-term subculture on agar plates containing macrolides.

We found that long-term macrolide exposure did not change the MICs of the antibiotics tested. Similarly, bacterial growth rate was also unchanged, consistent with the results of short-term macrolide exposure [29]. These results suggest that macrolides do not mediate direct antibacterial activity against $P$. aeruginosa. 

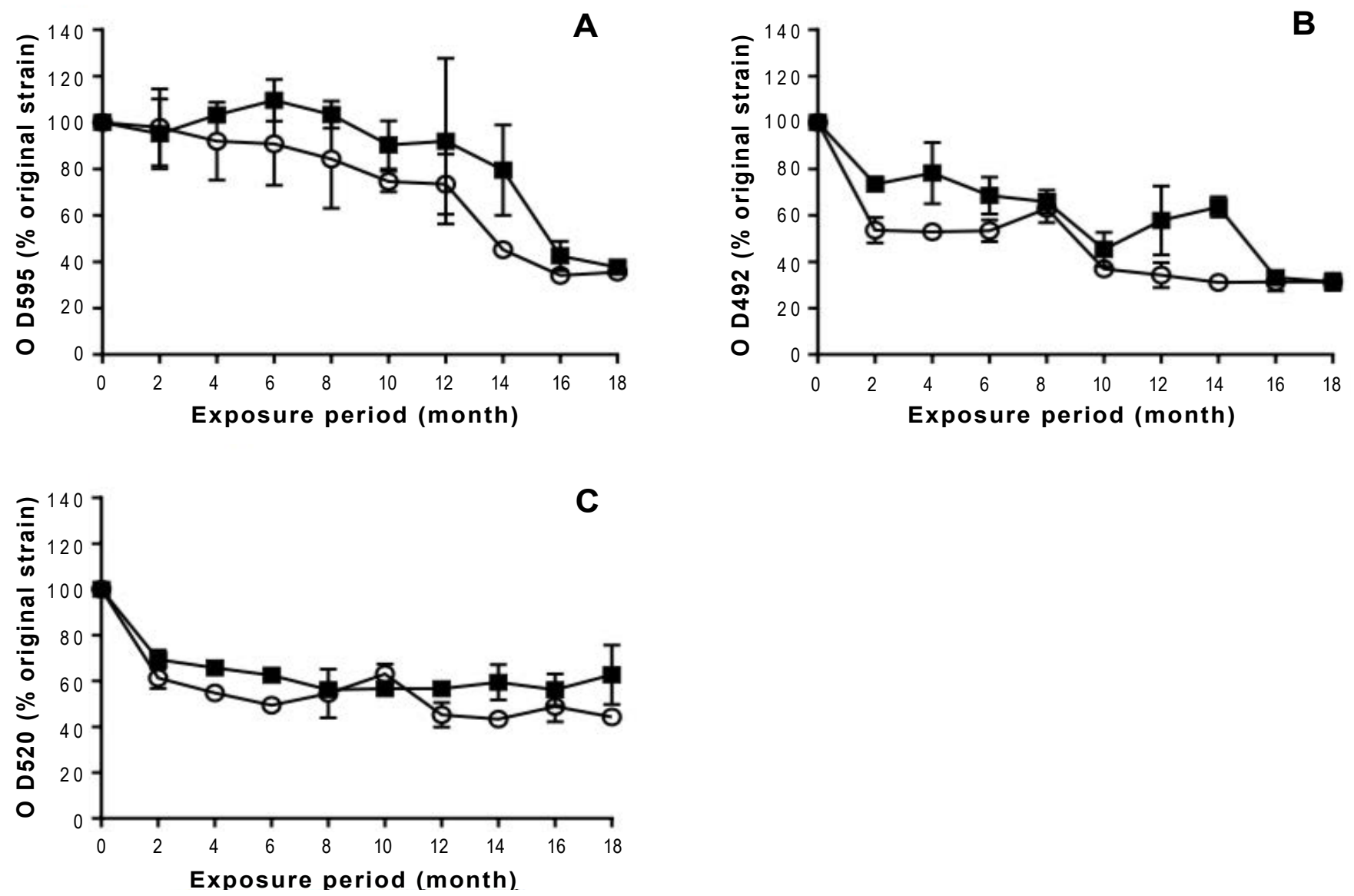

Figure 4 Virulence factors such as total protease (A), elastase (B), and pyocyanin (C) of PAO1 exposed to erythromycin (O) and clarithromycin (匹). Results were normalized to PAO1 with no macrolide exposure (original strain), and graphs display mean \pm SD of three independent experiments.

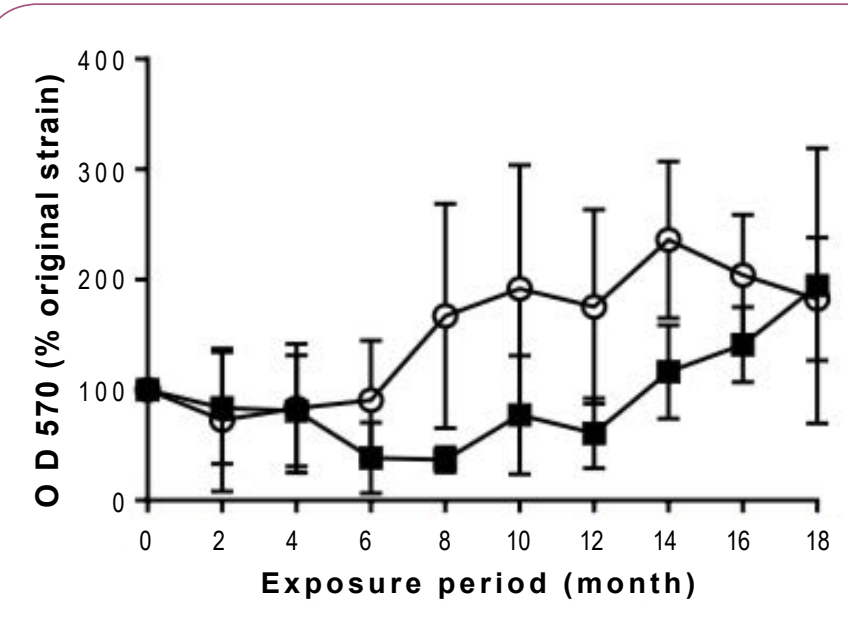

Figure 5 Analysis of biofilm formation by PAO1 exposed to erythromycin (O) and clarithromycin (ם). Results were normalized to PAO1 with no macrolide exposure (original strain), and the graphs show mean \pm SD of four independent experiments.

On the other hand, multiple virulence factors were inhibited in a time-dependent manner. The inhibition of swarming and twitching were consistent with the results of short-term macrolide exposure $[1,30]$. Swarming and twitching are involved in surface motility, bacterial adhesion, and microcolony formation [31,32]. It has been suggested that inhibition of swarming and twitching may gradually reduce these functions. In addition, we also observed inhibition of the production of rhamnolipids, biological surfactants associated with swarming [33], which is consistent with the report that culture on plates containing macrolides inhibited rhamnolipid production [17]. Our results suggest that inhibition of rhamnolipid production may be involved in the timedependent inhibition of swarming. Similarly, we confirmed that virulence factors such as total protease, elastase, and pyocyanin were inhibited in a time-dependent fashion. The observed inhibition of virulence factors was consistent with previous reports about the effects of short-term macrolide exposure $[14,15]$. Long-term macrolide exposure may gradually reduce the pathogenicity of $P$. aeruginosa via time-dependent inhibition of multiple virulence factors. As for the treatment period of macrolide long-term, low-dose therapy is recommended 2 years in Japan's guidelines [18]. However, there were few reports supporting appropriate treatment period of macrolide therapy in vitro or in vivo. The results of our research suggest that lowdose macrolide therapy for chronic airway infection should be 
administered for at least 2 months to achieve optimal effects against $P$. aeruginosa. The research results are the most useful data to determine the treatment period of macrolide therapy and we suggested the importance of the continuation of macrolide administration in clinical treatment.

Interestingly, several virulence factors were inhibited after 2 months of macrolide exposure. This timeframe is consistent with the period in which respiratory function and subjective symptoms were improved during long-term macrolide therapy $[34,35]$. Thus, inhibition of $P$. aeruginosa virulence factors after 2 months of macrolide therapy may be related to the improvement of respiratory function and subjective symptoms.

Our findings regarding biofilm formation were not concordant with a previous report of $P$. aeruginosa exposure to macrolides. The previous report suggested that macrolide treatment inhibited biofilm formation [16]. Our results showed a similar inhibition of biofilm formation until the 6 month of erythromycin exposure or the 12 month of clarithromycin exposure. However, prolongation

\section{References}

1 Wozniak DJ, Keyser R (2004) Effects of subinhibitory concentrations of macrolide antibiotics on Pseudomonas aeruginosa. Chest 125: 62S-69S

2 Iversen BG, Jacobsen T, Eriksen HM, Bukholm G, Melby KK, et al. (2007) An outbreak of Pseudomonas aeruginosa infection caused by contaminated mouth swabs. Clin Infect Dis 44: 794-801.

3 Yeung AT, Torfs EC, Jamshidi F, Bains M, Wiegand I, et al. (2009) Swarming of Pseudomonas aeruginosa is controlled by a broad spectrum of transcriptional regulators, including MetR. J Bacteriol 191: 5592-602.

4 Van Delden C, Iglewski BH (1998) Cell-to-cell signaling and Pseudomonas aeruginosa infections. Emerg Infect Dis 4: 551-560.

5 Gellatly SL, Hancock RE (2013) Pseudomonas aeruginosa: new insights into pathogenesis and host defenses. Pathog Dis 67: 159-173.

6 Sadikot RT, Blackwell TS, Christman JW, Prince AS (2005) Pathogenhost interactions in Pseudomonas aeruginosa pneumonia. Am J Respir Crit Care Med 171: 1209-1223.

7 Rao J, Damron FH, Basler M, Digiandomenico A, Sherman NE, et al. (2011) Comparisons of Two Proteomic Analyses of Non-Mucoid and Mucoid Pseudomonas aeruginosa Clinical Isolates from a Cystic Fibrosis Patient. Front Microbiol 2: 162.

8 Karatuna O, Yagci A (2010) Analysis of quorum sensing-dependent virulence factor production and its relationship with antimicrobial susceptibility in Pseudomonas aeruginosa respiratory isolates. Clin Microbiol Infect 16: 1770-1775.

9 Emerson J, Rosenfeld M, McNamara S, Ramsey B, Gibson RL (2002) Pseudomonas aeruginosa and other predictors of mortality and morbidity in young children with cystic fibrosis. Pediatr Pulmonol 34 91-100.

10 Fujii T, Kadota J, Kawakami K, Lida K, Shirai R, et al. (1995) Long term effect of erythromycin therapy in patients with chronic Pseudomonas aeruginosa infection. Thorax 50: 1246-1252. of macrolide exposure resulted in promotion of biofilm formation. These results indicate that long-term macrolide exposure, unlike short-term exposure, may promote biofilm formation.

We investigated for the first time $P$. aeruginosa subcultured on agar plates containing macrolides over an extended period of time. Our results suggest that long-term macrolide exposure inhibits multiple virulence factors in a time-dependent fashion. Those virulence factors are controlled by regulatory mechanisms such as quorum sensing, cyclic-di-GMP, and the GacS/GacA two component system [36]. It has been reported that quorum sensing is inhibited by macrolides [17]. Therefore, our results may be caused by macrolide-mediated inhibition of regulatory mechanisms. However, since the details of this result are not clear, there is room for further investigation.

\section{Disclosure}

We have no conflicts of interests to declare.

11 Kudoh S, Azuma A, Yamamoto M, Izumi T, Ando M (1998) Improvement of survival in patients with diffuse panbronchiolitis treated with low-dose erythromycin. Am J Respir Crit Care Med 157: 1829-1832.

12 Sakata K, Yajima H, Tanaka K, Sakamoto Y, Yamamoto K, et al. (1993) Erythromycin inhibits the production of elastase by Pseudomonas aeruginosa without affecting its proliferation in vitro. Am Rev Respir Dis 148: 1061-1065.

13 Kudoh S (1998) Erythromycin treatment in diffuse panbronchiolitis. Curr Opin Pulm Med 4: 116-121.

14 Molinari G, Guzmán CA, Pesce A, Schito GC (1993) Inhibition of Pseudomonas aeruginosa virulence factors by subinhibitory concentrations of azithromycin and other macrolide antibiotics. $J$ Antimicrob Chemother 31: 681-688.

15 Kita E, Sawaki M, Oku D, Hamuro A, Mikasa K, et al. (1991) Suppression of virulence factors of Pseudomonas aeruginosa by erythromycin. $J$ Antimicrob Chemother 27: 273-284.

16 Nagino K, Kobayashi H (1997) Influence of macrolides on mucoid alginate biosynthetic enzyme from Pseudomonas aeruginosa. Clin Microbiol Infect 3: 432-439.

17 Tateda K, Comte R, Pechere JC, Köhler T, Yamaguchi K, et al. (2001) Azithromycin inhibits quorum sensing in Pseudomonas aeruginosa. Antimicrob Agents Chemother 45: 1930-1933.

18 Mikasa K, Aoki N, Aoki Y, Abe S, Iwata S, et al. (2016) JAID/JSC Guidelines for the Treatment of Respiratory Infectious Diseases: The Japanese Association for Infectious Diseases/Japanese Society of Chemotherapy - The JAID/JSC Guide to Clinical Management of Infectious Disease/Guideline-preparing Committee Respiratory Infectious Disease WG. J Infect Chemother 22: S1-S65.

19 Medicines and Healthcare products Regulatory Agency (2007) Safeguarding public health; Erythromycin 250 mg Capsules, PL 10622/0148. London: MHRA 121

20 Medicines and Healthcare products Regulatory Agency (2008) Safeguarding public health; Clarithromycin 250 and $500 \mathrm{mg}$ Film Coated Tablets, PL 20897/0001-2. London: MHRA 22: 186. 
21 Clinical and Laboratory Standards Institute (2015) Performance standards for antimicrobial susceptibility testing: 25th informational supplement. CLSI M 100-S25.

22 Rashid MH, Kornberg A (2000) Inorganic polyphosphate is needed for swimming, swarming, and twitching motilities of Pseudomonas aeruginosa. Proc Natl Acad Sci USA 97: 4885-4890.

23 Maniatis T, Fritsch EF, Sambrook J (1982) Molecular cloning: a laboratory manual. NY: Cold Spring Harbor Laboratory 14: 195.

24 Köhler T, van Delden C, Curty LK, Hamzehpour MM, Pechere JC (2001) Overexpression of the MexEF-OprN multidrug efflux system affects cell-to-cell signaling in Pseudomonas aeruginosa. J Bacteriol 183: 5213-5222.

25 Head NE, Yu H (2004) Cross-sectional analysis of clinical and environmental isolates of Pseudomonas aeruginosa: biofilm formation, virulence, and genome diversity. Infect Immun 72: 133-144.

26 Kumar L, Chhibber S, Kumar R, Harjai K (2015) Zingerone silences quorum sensing and attenuates virulence of Pseudomonas aeruginosa. Fitoterapia 102: 84-95.

27 El-Mowafy SA, Abd El Galil KH, El-Messery SM, Shaaban MI (2014) Aspirin is an efficient inhibitor of quorum sensing, virulence and toxins in Pseudomonas aeruginosa. Microb Pathog 74: 25-32.

28 Essar DW, Eberly L, Hadero A, Crawford IP (1990) Identification and characterization of genes for a second anthranilate synthase in Pseudomonas aeruginosa: interchangeability of the two anthranilate synthases and evolutionary implications. J Bacteriol 172: 884-900.
29 Mizukane R, Hirakata Y, Kaku M, Ishii Y, Furuya N, et al. (1994) Comparative in vitro exoenzyme-suppressing activities of azithromycin and other macrolide antibiotics against Pseudomonas aeruginosa. Antimicrob Agents Chemother 38: 528-533.

30 Kawamura-Sato K, linuma Y, Hasegawa T, Horii T, Yamashino T, et al. (2000) Effect of subinhibitory concentrations of macrolides on expression of flagellin in Pseudomonas aeruginosa and Proteus mirabilis. Antimicrob Agents Chemother 44: 2869-2872.

31 O'May C, Tufenkji N (2011) The swarming motility of Pseudomonas aeruginosa is blocked by cranberry proanthocyanidins and other tannin-containing materials. Appl Environ Microbiol 77: 3061-3067.

32 O'Toole GA, Kolter R (1998) Flagellar and twitching motility are necessary for Pseudomonas aeruginosa biofilm development. Mol Microbiol 30: 295-304.

33 Köhler T, Curty LK, Barja F, van Delden C, Pechère JC (2000) Swarming of Pseudomonas aeruginosa is dependent on cell-to-cell signaling and requires flagella and pili. J Bacteriol 182: 5990-5996.

34 Kadota J, Mukae H, Ishii H, Nagata T, Kaida H, et al. (2003) Longterm efficacy and safety of clarithromycin treatment in patients with diffuse panbronchiolitis. Respir Med 97: 844-850.

35 Lin X, Lu J, Yang M, Dong BR (2015) Macrolides for diffuse panbronchiolitis. Cochrane Database Syst Rev 1: CD007716.

36 Jimenez PN, Koch G, Thompson JA, Xavier KB, Cool RH, et al. (2012) The multiple signaling systems regulating virulence in Pseudomonas aeruginosa. Microbiol Mol Biol Rev 76: 46-65. 